\title{
Irregular Tilings of Regular Polygons with Similar Triangles
}

\author{
Miklos Laczkovich ${ }^{1}$
}

Received: 10 November 2019 / Revised: 29 October 2020 / Accepted: 4 March 2021 /

Published online: 10 May 2021

(c) The Author(s) 2021

\begin{abstract}
We say that a triangle $T$ tiles a polygon $A$, if $A$ can be dissected into finitely many nonoverlapping triangles similar to $T$. We show that if $N>42$, then there are at most three nonsimilar triangles $T$ such that the angles of $T$ are rational multiples of $\pi$ and $T$ tiles the regular $N$-gon. A tiling into similar triangles is called regular, if the pieces have two angles, $\alpha$ and $\beta$, such that at each vertex of the tiling the number of angles $\alpha$ is the same as that of $\beta$. Otherwise the tiling is irregular. It is known that for every regular polygon $A$ there are infinitely many triangles that tile $A$ regularly. We show that if $N>10$, then a triangle $T$ tiles the regular $N$-gon irregularly only if the angles of $T$ are rational multiples of $\pi$. Therefore, the number of triangles tiling the regular $N$-gon irregularly is at most three for every $N>42$.
\end{abstract}

Keywords Tilings with triangles $\cdot$ Regular polygons $\cdot$ Regular and irregular tilings

Mathematical Subject Classification 52C20

\section{Introduction}

Dissections of regular polygons appear in several popular puzzles (see [1]). Some of these dissections, such as Langford's dissections of the regular pentagon [7], Freese's dissection of the regular octagon [1, Fig. 17.1], or Kürschák's dissection of the regular 12-gon [2, Fig. 2.6.4], consist of triangles of two different shapes.

Editor in Charge: János Pach

The author was supported by the Hungarian National Foundation for Scientific Research, Grant No. K124749.

Miklos Laczkovich

miklos.laczkovich@gmail.com

1 Department of Analysis, Eötvös Loránd University (ELTE), Pázmány Péter sétány 1/c, 1117

Budapest, Hungary 
In this paper we consider dissections of the regular polygons using triangles of one single shape but not necessarily of the same size. What we are interested in is the existence of tilings, independently of the rearrangement of the pieces (which is the usual motivation for the puzzles mentioned). We confine our attention to triangles having angles that are rational multiples of $\pi$. Our aim is to show that if $N$ is large enough, then there are at most three nonsimilar triangles $T$ in this class such that the regular $N$-gon can be dissected into similar copies of $T$.

\subsection{Main Results}

By a dissection (or tiling) of a polygon $A$ we mean a decomposition of $A$ into finitely many nonoverlapping polygons. No other conditions are imposed on the tilings. In particular, it is allowed that two pieces have a common boundary point, but do not have a common side. We say that a triangle $T$ tiles a polygon $A$, if $A$ can be dissected into finitely many nonoverlapping triangles similar to $T$. Our main result is the following.

Theorem 1.1 Suppose that a triangle with angles $\alpha, \beta, \gamma$ tiles the regular $N$-gon, where $N \geq 25$ and $N \neq 30,42$. If $\alpha, \beta, \gamma$ are rational multiples of $\pi$, then, after $a$ suitable permutation of $\alpha, \beta, \gamma$, one of the following statements is true:

(i) $\alpha=(N-2) \pi / N$ and $\beta=\gamma=\pi / N$,

(ii) $\alpha=\beta=(N-2) \pi /(2 N)$ and $\gamma=2 \pi / N$, or

(iii) $\alpha=(N-2) \pi /(2 N), \beta=\pi / N$, and $\gamma=\pi / 2$.

Let $R_{N}$ and $\delta_{N}$ denote the regular $N$-gon and its angle; that is, let $\delta_{N}=(N-2) \pi / N$. Connecting the center of $R_{N}$ with the vertices of $R_{N}$ we obtain a dissection of $R_{N}$ into $N$ congruent isosceles triangles with angles listed in (ii). Bisecting each of these triangles into two right angled triangles, we get a dissection of $R_{N}$ into $2 N$ congruent triangles with angles listed in (iii).

Thus the triangles with angles listed in (ii) and (iii) tile $R_{N}$, even with congruent copies. This is also true for the triangle with angles listed in (i) if $N=3$, 4, or 6 . (As for $N=6$, see Fig. 1.) If $N$ is different from 3, 4, or 6, then dissections of $R_{N}$ with congruent copies of a triangle with angles $\alpha=\delta_{N}$ and $\beta=\gamma=\pi / N$ do not exist (see [5, Lem. 3.5]). It is not clear, however, if $R_{N}$ can be dissected into similar triangles of angles $\alpha=\delta_{N}$ and $\beta=\gamma=\pi / N$ for every $N$. If $N=5,8,10$, or 12, then such tilings exist (see [6]).

Suppose there is a tiling of $R_{N}$ with triangles of angles $\alpha, \beta, \gamma$, and let $V_{1}, \ldots, V_{M}$ be an enumeration of the vertices of the tiles such that $V_{1}, \ldots, V_{N}$ are the vertices of $R_{N}$. Let $p_{j}, q_{j}, r_{j}$ denote the number of angles $\alpha, \beta$, resp. $\gamma$ meeting at the vertex $V_{j}$. Then $p_{j}, q_{j}, r_{j}, j=1, \ldots, M$, are nonnegative integers such that

$$
p_{j} \alpha+q_{j} \beta+r_{j} \gamma=\sigma_{j}, \quad j=1, \ldots, M,
$$

where $\sigma_{j}=\delta_{N}$ if $1 \leq j \leq N$, and $\sigma_{j}=\pi$ or $2 \pi$ depending on whether $V_{j}$ is on the boundary or in the interior of $R_{N}$ if $N<j \leq M$. The integers $p_{j}, q_{j}, r_{j}$ must also satisfy 


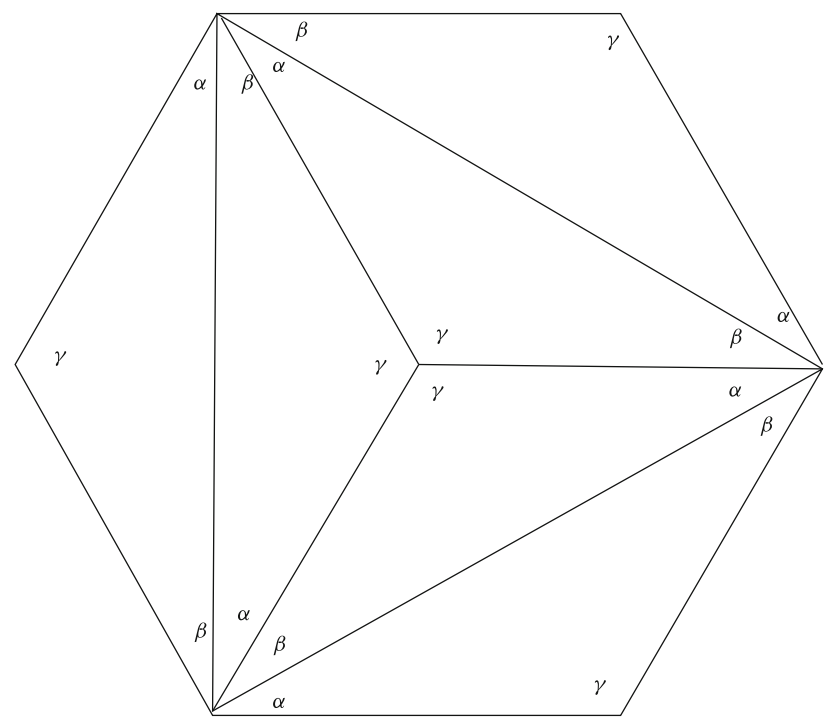

Fig. 1 A (regular) tiling of $R_{6}$

$$
\sum_{j=1}^{M} p_{j}=\sum_{j=1}^{M} q_{j}=\sum_{j=1}^{M} r_{j},
$$

since each sum must be equal to the number of tiles. We call (1) the equation at the vertex $V_{j}$. Theorem 1.1 will be proved through the following results.

Theorem 1.2 Suppose a tiling of $R_{N}$ with triangles of angles $\alpha, \beta, \gamma$ is given, where $\alpha, \beta, \gamma$ are rational multiples of $\pi$. If $N \neq 6$, then each angle of $R_{N}$ is packed with at most two tiles.

Theorem 1.3 Suppose a tiling of $R_{N}$ with triangles of angles $\alpha, \beta, \gamma$ is given, where $\alpha, \beta, \gamma$ are rational multiples of $\pi$ and $N>6$. Then the equations at the vertices $V_{1}, \ldots, V_{N}$ are the same. More precisely, after a suitable permutation of $\alpha, \beta, \gamma$, one of the following is true:

(i) The equation at every vertex of $R_{N}$ is $\alpha=\delta_{N}$.

(ii) The equation at every vertex of $R_{N}$ is $\alpha+\beta=\delta_{N}$.

(iii) The equation at every vertex of $R_{N}$ is $2 \alpha=\delta_{N}$.

Theorem 1.4 Suppose a tiling of $R_{N}$ with triangles of angles $\alpha, \beta, \gamma$ is given, where $\alpha, \beta, \gamma$ are rational multiples of $\pi$ and $N>5$. If the equation at every vertex of $R_{N}$ is $\alpha=\delta_{N}$, then we have $\beta=\gamma=\pi / N$.

Theorem 1.5 Suppose a tiling of $R_{N}$ with triangles of angles $\alpha, \beta, \gamma$ is given, where $\alpha, \beta, \gamma$ are rational multiples of $\pi$ and $N>10$. If the equations at the vertices of $R_{N}$ are $\alpha+\beta=\delta_{N}$, then we have $\alpha=\beta=\delta_{N} / 2$ and $\gamma=2 \pi / N$. 


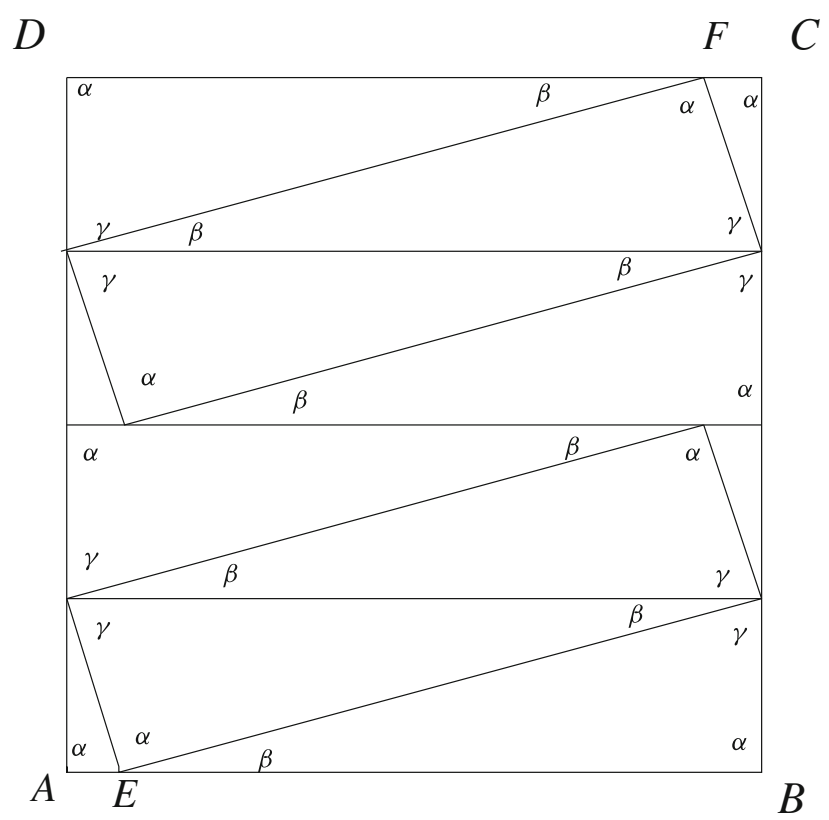

Fig. 2 A tiling of the square with $\alpha=\delta_{4}$

Theorem 1.6 Suppose a tiling of $R_{N}$ with triangles of angles $\alpha, \beta, \gamma$ is given, where $\alpha, \beta, \gamma$ are rational multiples of $\pi$ and $N \geq 25$ with $N \neq 30,42$. If the equations at the vertices of $R_{N}$ are $2 \alpha=\delta_{N}$, then we have either $\alpha=\gamma=\delta_{N} / 2$ and $\beta=2 \pi / N$, or $\alpha=\delta_{N} / 2, \beta=\pi / N$, and $\gamma=\pi / 2$.

It is clear that Theorem 1.1 follows from Theorems 1.3-1.6.

Remark 1.7 As Fig. 1 shows, the statements of Theorems 1.2 and 1.3 are not true for $N=6$.

The lower bound in Theorem 1.4 is also sharp, moreover, the statement of the theorem is false for every $N \leq 5$. One can show, using the ideas of [6] that the triangle with angles $\alpha=6 \pi / 10, \beta=\pi / 10$, and $\gamma=3 \pi / 10$ tiles $R_{5}$ so that the equation at each vertex of $R_{5}$ is $\alpha=\delta_{5}$ (but $\beta \neq \gamma$ ).

As for $N=4$, Fig. 2 shows a tiling of the square $A B C D$ with 12 right triangles of angles $\alpha=\pi / 2, \beta=\pi / 12$, and $\gamma=5 \pi / 12$. If the side length of the square is 4 then we have $\overline{A E}=\overline{F C}=2-\sqrt{3}$ and $\overline{E B}=\overline{D F}=2+\sqrt{3}$. Note that in this tiling $\alpha=\delta_{4}$ at each vertex of the square, but $\beta \neq \gamma$.

It is easy to see that the regular triangle can be decomposed into eight congruent triangles with angles $\alpha=\pi / 3, \beta=\pi / 6$, and $\gamma=\pi / 2$ such that the equation at each vertex of $R_{3}$ is $\alpha=\delta_{3}$.

As for the sharpness of the bounds appearing in Theorems 1.5 and 1.6 we refer to Remark 1.11 below. 


\subsection{Regular and Irregular Tilings}

A tiling into similar triangles is called regular, if the pieces have two angles, $\alpha$ and $\beta$, such that at each vertex $V$ of any of the tiles, the number of tiles having angle $\alpha$ at $V$ is the same as the number of tiles having angle $\beta$ at $V$. Otherwise the tiling is irregular. It is known that the number of triangles that tile a given polygon irregularly is always finite (see [4, Thm. 4]). On the other hand, for every $N \geq 3$ there are infinitely many triangles that tile the regular $N$-gon regularly (see [4, Thm. 2]).

The problem of listing all triangles that tile a given polygon is difficult; it is unsolved even for the regular triangle. In fact, the problem is solved only for the square; see $[3,8]$. (See also [5], where the tilings of convex polygons with congruent triangles are considered.) As for irregular tilings of $R_{N}, N>10$, we have the following corollary of Theorems 1.3-1.5.

Theorem 1.8 Suppose a triangle $T$ with angles $\alpha, \beta, \gamma$ tiles $R_{N}$, where $N>10$. Then there is an irregular tiling of $R_{N}$ with pieces similar to $T$ if and only if $\alpha, \beta, \gamma$ are rational multiples of $\pi$.

Proof Suppose there is an irregular tiling of $R_{N}$ with pieces similar to $T$. Let $V_{1}, \ldots, V_{M}$ denote the vertices of the tiles, where $M \geq N$ and $V_{1}, \ldots, V_{N}$ are the vertices of $R_{N}$. If the number of angles $\alpha, \beta, \gamma$ meeting at $V_{j}$ is $p_{j}, q_{j}, r_{j}$, respectively, then (1) and (2) are satisfied. The irregularity of the tiling means that there are indices $i, j, k$ such that $p_{i} \neq q_{i}, p_{j} \neq r_{j}$, and $q_{k} \neq r_{k}$.

Consider all systems of nonnegative integers $s_{j}, t_{j}, u_{j}, j=1, \ldots, m, m \geq N$, such that

(i) $s_{j} \alpha+t_{j} \beta+u_{j} \gamma=\sigma_{j}, j=1, \ldots, m$, where $\sigma_{j}=\delta_{N}$ if $1 \leq j \leq N$ and $\sigma_{j}=\pi$ or $2 \pi$ if $N<j \leq m$,

(ii) $\sum_{j=1}^{m} s_{j}=\sum_{j=1}^{m} t_{j}=\sum_{j=1}^{m} u_{j}$, and

(iii) there are indices $i, j, k$ such that $s_{i} \neq t_{i}, s_{j} \neq u_{j}$, and $t_{k} \neq u_{k}$.

Fix a system with these properties such that the value from (ii) is minimal. By [4, Lem. 10], these conditions imply that there are indices $i<j$ such that the determinant

$$
D_{i j}=\left|\begin{array}{ccc}
1 & 1 & 1 \\
s_{i} & t_{i} & u_{i} \\
s_{j} & t_{j} & u_{j}
\end{array}\right|
$$

is nonzero. Then the corresponding system of equations

$$
\begin{aligned}
\alpha+\beta+\gamma & =\pi, \\
s_{i} \alpha+t_{i} \beta+u_{i} \gamma & =\sigma_{i} \\
s_{j} \alpha+t_{j} \beta+u_{j} \gamma & =\sigma_{j}
\end{aligned}
$$

determines $\alpha, \beta, \gamma$. Applying Cramer's rule, we find that $\alpha, \beta, \gamma$ are rational multiples of $\pi$. 
Next let $\alpha, \beta, \gamma$ be rational multiples of $\pi$. Since $N>10$, one of (i)-(iii) of Theorem 1.3 holds. If (i) or (ii) holds, then it follows from Theorems 1.4 and 1.5 that $T$ is isosceles. Suppose $\alpha=\beta$, and consider a tiling of $R_{N}$ with pieces similar to $T$. If the tiling is irregular, we are done. If, however, it is regular, then changing the labels $\alpha$ and $\beta$ in one of the pieces we obtain an irregular tiling.

Now suppose that (iii) of Theorem 1.3 holds. We prove that in this case every tiling with similar copies of $T$ must be irregular. Suppose this is not true, and consider a regular tiling. Let (1) be the equation at the vertex $V_{j}, j=1, \ldots, M$. We put $v_{j}=\sigma_{j} / \pi$, then $v_{j}=1$ or 2 for every $N<j \leq M$. Since the equation at each vertex of $R_{N}$ is $2 \alpha=\delta_{N}$, it follows that $q_{j}=r_{j}$ for every $j$. Then there must be an equation with $p_{j}<q_{j}=r_{j}$, since in the equations at the vertices of $R_{N}$ we have $p_{j}>q_{j}=0$. For this equation we have

$$
\begin{aligned}
\left(q_{j}-p_{j}\right)(\beta+\gamma) & =\left(p_{j} \alpha+q_{j} \beta+r_{j} \gamma\right)-p_{j}(\alpha+\beta+\gamma) \\
& =v_{j} \pi-p_{j} \pi=\left(v_{j}-p_{j}\right) \pi,
\end{aligned}
$$

hence $\left(q_{j}-p_{j}\right)(1 / 2+1 / N)=v_{j}-p_{j}$ and $(N+2)\left(q_{j}-p_{j}\right)=2 N\left(v_{j}-p_{j}\right)$. Since $q_{j}-p_{j}$ is a positive integer, we have $(N+2) \mid 2 N\left(v_{j}-p_{j}\right)$ and $(N+2) \mid 4\left(v_{j}-p_{j}\right)$. Now $v_{j}-p_{j}$ is positive, since $(N+2)\left(q_{j}-p_{j}\right)>0$. Then $0<v_{j}-p_{j} \leq 2$, $0<4\left(v_{j}-p_{j}\right) \leq 8$, and thus $(N+2) \mid 4\left(v_{j}-p_{j}\right)$ implies $N \leq 6$, which is impossible.

Comparing Theorem 1.8 with Theorem 1.1 we obtain the following.

Corollary 1.9 If $N>42$, then there are at most three triangles that tile the regular $N$-gon irregularly.

\subsection{Conditions (K) and (E)}

The main tool in the proof of Theorems 1.2-1.6 is the next result.

Lemma 1.10 Suppose $R_{N}$ can be dissected into finitely many triangles with angles $\alpha=(a / n) \pi, \beta=(b / n) \pi, \gamma=(c / n) \pi$, where $a, b, c, n$ are positive integers with $a+b+c=n$. Let the equation at the vertices of $R_{N}$ be $p_{j} \alpha+q_{j} \beta+r_{j} \gamma=\delta_{N}$, $j=1, \ldots, N$. If $k$ is prime to $n N$ and $\{k / N\}<1 / 2$, then we have

$$
\begin{gathered}
\left\{\frac{k a}{n}\right\}+\left\{\frac{k b}{n}\right\}+\left\{\frac{k c}{n}\right\}=1, \\
p_{j}\left\{\frac{k a}{n}\right\}+q_{j}\left\{\frac{k b}{n}\right\}+r_{j}\left\{\frac{k c}{n}\right\}=1-2\left\{\frac{k}{N}\right\}
\end{gathered}
$$

for every $j=1, \ldots, N$.

We say that the angles $\alpha=(a / n) \pi, \beta=(b / n) \pi, \gamma=(c / n) \pi$ satisfy condition $(\mathrm{K})$, if the conclusion of the lemma above holds; that is, if (3) and (4) hold true for every $k$ such that $\operatorname{gcd}(k, n N)=1$ and $\{k / N\}<1 / 2$. As we shall see in the next section, condition $(\mathrm{K})$ is deduced from the properties of conjugate tilings. 
If a tiling exists with triangles of angles $\alpha, \beta, \gamma$, then the angles have to satisfy another necessary condition: there must exist nonnegative integers $p_{j}, q_{j}, r_{j}, j=$ $1, \ldots, M, M \geq N$, such that (1) and (2) hold. We say that the angles $\alpha, \beta, \gamma$ satisfy condition (E), if there are nonnegative integers $p_{j}, q_{j}, r_{j}$ satisfying (1) and (2).

In the proof of Theorems 1.2-1.6 we only use conditions (K) and (E) on the angles $\alpha, \beta, \gamma$. In fact, we are not aware of any other necessary condition that must be satisfied by the angles of a tiling, if they are rational multiples of $\pi$. Perhaps it would be hasty to conjecture that whenever the angles of a triangle satisfy conditions $(\mathrm{K})$ and $(\mathrm{E})$, then a tiling must exist. Still, it should be remarked that tilings of $R_{N}$ with triangles of angles $\alpha=\delta_{N}$ and $\beta=\gamma=\pi / N$ were found for $N=5,8,10$, and 12 in [6]. In this context we also mention Szegedy's remarkable tilings of the square with right triangles, found ten years after the necessary conditions were established [8].

Remark 1.11 We do not know if the lower bounds in Theorems 1.5 and 1.6 are sharp or not. We show, however, that if we only use conditions $(\mathrm{K})$ and $(\mathrm{E})$, then these bounds cannot be improved. As for Theorem 1.5, consider the triangle $T$ with angles

$$
(\alpha, \beta, \gamma)=\left(\frac{7 \pi}{10}, \frac{\pi}{10}, \frac{2 \pi}{10}\right) .
$$

Then the existence of a tiling of $R_{10}$ with similar copies of $T$ cannot be disproved by only using conditions (K) and (E). Suppose that the equation at each vertex of $R_{10}$ is $\alpha+\beta=\delta_{10}$. Then condition (K) is satisfied. Indeed, the only $k$ with $1<k<10$, $\operatorname{gcd}(k, 10)=1$, and $\{k / 10\}<1 / 2$ is $k=3$, and it is easy to check that both (3) and (4) are satisfied if $(a / n, b / n, c / n)=(7 / 10,1 / 10,2 / 10)$ and $k=3$. Condition (E) is also satisfied: take 10 equations $\alpha+\beta=\delta_{10}$ and an equation $10 \gamma=2 \pi$.

In the case of Theorem 1.6, consider the triangle with angles

$$
(\alpha, \beta, \gamma)=\left(\frac{20 \pi}{42}, \frac{10 \pi}{42}, \frac{12 \pi}{42}\right)
$$

and let the equation at each vertex of $R_{42}$ be $2 \alpha=\delta_{42}$. Then condition $(\mathrm{K})$ is satisfied. Indeed, if $1<k<42, \operatorname{gcd}(k, 42)=1$, and $\{k / 42\}<1 / 2$, then $k$ is one of $5,11,13,17,19$. It is easy to check that both (3) and (4) are satisfied if $(a / n, b / n, c / n)=(20 / 42,10 / 42,12 / 42)$ and if $k$ is any of these values. Condition (E) is also satisfied: take 42 equations $2 \alpha=\delta_{42}, 8$ equations $7 \gamma=2 \pi$, and 28 equations $3 \beta+\gamma=\pi$.

Similarly, if $N=30$, then the triple

$$
\left(\frac{14 \pi}{30}, \frac{6 \pi}{30}, \frac{10 \pi}{30}\right)
$$

satisfies both conditions (K) and (E). As for the latter, take 30 equations $2 \alpha=\delta_{30}, 20$ equations $3 \gamma=\pi$, and 12 equations $5 \beta=\pi$. 


\subsection{Further Lemmas}

Since condition $(\mathrm{K})$ is of arithmetical nature, it can be expected that in the arguments involving condition $(\mathrm{K})$ we need some facts from elementary number theory. These facts are collected in the next lemmas. Their proofs, being independent of the rest of the paper, are postponed to the last three sections.

Lemma 1.12 Let $a, n, N, N^{\prime}$ be positive integers such that $\operatorname{gcd}(a, n)=1$ and $\operatorname{gcd}\left(N, N^{\prime}\right)=1$. Then one of the following statements is true.

(i) There exists an integer $k$ such that $\operatorname{gcd}(k, n N)=1, k \equiv N^{\prime}(\bmod N)$, and $\{k a / n\} \geq 1 / 3$.

(ii) $N$ is odd and $n \mid 2 N$.

(iii) $N$ is even and $n \mid N$.

Lemma 1.13 Let $a, b, n, N$ be positive integers and $p, q$ be nonnegative integers such that $a+b<n, N \geq 3, N \neq 6$, and

$$
p\left\{\frac{k a}{n}\right\}+q\left\{\frac{k b}{n}\right\}=1-2\left\{\frac{k}{N}\right\}
$$

for every integer $k$ satisfying $\operatorname{gcd}(k, n N)=1$ and $\{k / N\}<1 / 2$. Then we have $p+q \leq 2$.

Note that Theorem 1.2 is a consequence of Lemmas 1.10 and 1.13. Indeed, suppose a tiling of $R_{N}$ with triangles of angles $\alpha, \beta, \gamma$ is given, where $\alpha, \beta, \gamma$ are rational multiples of $\pi$. Then $\alpha=(a / n) \pi, \beta=(b / n) \pi$, and $\gamma=(c / n) \pi$, where $a, b, c, n$ are positive integers with $a+b+c=n$. Let (1) be the equation at the vertex $V_{j}$, $j=1, \ldots, M$. By Lemma 1.10, we have (4) for every $j=1, \ldots, N$, whenever $k$ is prime to $n N$ and $\{k / N\}<1 / 2$.

Let $j \leq N$ be fixed. Then $\min \left(p_{j}, q_{j}, r_{j}\right)=0$, since $\sigma_{j}=\delta_{N}<\pi$. By symmetry we may assume $r_{j}=0$. Then (5) holds with $p=p_{j}$ and $q=q_{j}$ for every $k$ such that $\operatorname{gcd}(k, n N)=1$ and $\{k / N\}<1 / 2$. If $N \neq 6$ then, by Lemma 1.13, we obtain that $p_{j}+q_{j} \leq 2$. Therefore, the number of angles meeting at the vertex $V_{j}$ is $p_{j}+q_{j} \leq 2$; that is, the angle of $R_{N}$ at the vertex $V_{j}$ is packed with at most two tiles.

Lemma 1.14 (i) For every even integer $N \geq 26$ there are integers $k, k^{\prime}$ such that $N / 4<k, k^{\prime}<N / 2, \operatorname{gcd}(k, N)=\operatorname{gcd}\left(k^{\prime}, N\right)=1, k \equiv 1(\bmod 4)$, and $k^{\prime} \equiv 3$ $(\bmod 4)$.

(ii) For every $N \geq 43$ there exists an integer $k$ such that $N / 6<k<N / 4$ and $\operatorname{gcd}(k, 2 N)=1$.

The following simple observation will be used frequently.

Proposition 1.15 Let $u, v, n$ be nonzero integers. If $\operatorname{gcd}(u, v)=1$, then there exists an integer $j$ such that $u+j v$ is prime to $n$.

Proof Let $j$ be the product of those primes that divide $n$ but do not divide $u$. (We put $j=1$ if there is no such prime.) Then every prime divisor of $n$ divides exactly one of $u$ and $j v$, and thus $\operatorname{gcd}(u+j v, n)=1$. 
The paper is organised as follows. In the next five sections we prove Lemma 1.10 and Theorems 1.3-1.6, in this order. Then we prove Lemmas 1.12-1.14 in Sects. 7-9.

\section{Proof of Lemma 1.10}

Suppose $R_{N}$ is tiled with the triangles $\Delta_{1}, \ldots, \Delta_{t}$ with angles $\alpha=(a / n) \pi, \beta=$ $(b / n) \pi, \gamma=(c / n) \pi$, where $a, b, c, n$ are positive integers with $a+b+c=n$. Let the vertices of $R_{N}$ be the $N^{\text {th }}$ roots of unity; that is, let $V_{j}=e^{2 \pi j i / N}$ for every $j=$ $0, \ldots, N-1$. First we assume that $4 N \mid n$. Let $\zeta$ denote the first $n^{\text {th }}$ root of unity, and let $F$ denote the field of real elements of the cyclotomic field $\mathbb{Q}(\zeta)$. Then the coordinates of the vertices of $R_{N}$ belong to $F$, since $\cos 2 j \pi / N=\left(\zeta^{n j / N}+\zeta^{-n j / N}\right) / 2$ and $\sin 2 j \pi / N=\left(\zeta^{n j / N}-\zeta^{-n j / N}\right) /\left(2 \zeta^{n / 4}\right)$ for every integer $j$. Also, $\cot \alpha, \cot \beta, \cot \gamma$ belong to $F$, since

$$
\cot \frac{j \pi}{n}=\frac{e^{(j / n) \pi i}+e^{-(j / n) \pi i}}{e^{(j / n) \pi i}-e^{-(j / n) \pi i}} \cdot \zeta^{n / 4}=\frac{\zeta^{j}+1}{\zeta^{j}-1} \cdot \zeta^{n / 4}
$$

for every $j$. In [3, Thm. 1] it is proved that if a polygon $P$ is decomposed into the non-overlapping triangles $\Delta_{1}, \ldots, \Delta_{t}$, then the coordinates of the vertices of each $\Delta_{j}$ belong to the field generated by the coordinates of the vertices of $P$ and the cotangents of the angles of the triangles $\Delta_{j}$. In our case this implies that the coordinates of the vertices of the triangles $\Delta_{j}$ belong to $F$.

Let $k$ be an integer prime to $n$, and let $\phi: \mathbb{Q}(\zeta) \rightarrow \mathbb{C}$ be the isomorphism of $\mathbb{Q}(\zeta)$ satisfying $\phi(\zeta)=\zeta^{k}$. Then $\phi$ commutes with complex conjugation, and thus $\phi$ restricted to $F$ is also an isomorphism. It is easy to check that

$$
\phi\left(\cot \frac{j \pi}{n}\right)=(-1)^{(k-1) / 2} \cot \frac{k j \pi}{n}
$$

for every integer $j$. We define $\Phi(x, y)=(\phi(x), \phi(y))$ for every $x, y \in F$. Then $\Phi$ is a collineation defined on $F \times F$. In particular, $\Phi$ is defined on the set of vertices of the tiles $\Delta_{j}, j=1, \ldots, t$. We denote by $\Delta_{j}^{\prime}$ the triangle with vertices $\Phi\left(V_{j, 1}\right), \Phi\left(V_{j, 2}\right), \Phi\left(V_{j, 3}\right)$, where $V_{j, 1}, V_{j, 2}, V_{j, 3}$ are the vertices of $\Delta_{j}$.

Let the points $x_{i}=\left(a_{i}, b_{i}\right), i=1,2,3$, be the vertices of a triangle $\Delta$, where $a_{i}, b_{i} \in F, i=1,2,3$, and let $\Delta^{\prime}$ denote the triangle with vertices $\Phi\left(x_{i}\right), i=1,2,3$. Let $\alpha_{i}$ and $\alpha_{i}^{\prime}, i=1,2,3$, denote the angles of $\Delta$ and of $\Delta^{\prime}$, respectively. A simple computation shows that $\cot \alpha_{i}^{\prime}=\varepsilon \phi\left(\cot \alpha_{i}\right)$ holds for $i=1,2,3$, where $\varepsilon=1$ if the map $\Phi$ does not change the orientation of $\Delta$, and $\varepsilon=-1$ otherwise (see [3, Lem. 6]).

Put $\varepsilon_{j}=1$ if $\Phi$ does not change the orientation of $\Delta_{j}$ and $\varepsilon_{j}=-1$ otherwise. If the angles of $\Delta_{j}^{\prime}$ are $\alpha_{j}^{\prime}, \beta_{j}^{\prime}, \gamma_{j}^{\prime}$, then, by the result above, we have

$$
\cot \alpha_{j}^{\prime}=\varepsilon_{j} \cdot \phi(\cot \alpha)=\varepsilon_{j} \cdot(-1)^{(k-1) / 2} \cdot \cot \frac{k a \pi}{n}
$$


and, similarly,

$$
\cot \beta_{j}^{\prime}=\varepsilon_{j} \cdot(-1)^{(k-1) / 2} \cdot \cot \frac{k b \pi}{n}, \quad \cot \gamma_{j}^{\prime}=\varepsilon_{j} \cdot(-1)^{(k-1) / 2} \cdot \cot \frac{k c \pi}{n} .
$$

Note that at least two of the numbers $\cot \alpha_{j}^{\prime}, \cot \beta_{j}^{\prime}, \cot \gamma_{j}^{\prime}$ are positive for every $j$. Since the integers $a, b, c, n, k$ are fixed, this implies that the value of $\varepsilon_{j}$ is the same for every $j=1, \ldots, t$. Therefore, the orientation of the triangles $\Delta_{j}^{\prime}$ is the same, and the angles of each $\Delta_{j}^{\prime}$ are

$$
\alpha^{\prime}=\left\{\frac{k a}{n}\right\} \pi, \quad \beta^{\prime}=\left\{\frac{k b}{n}\right\} \pi, \quad \gamma^{\prime}=\left\{\frac{k c}{n}\right\} \pi
$$

if $\varepsilon \cdot(-1)^{(k-1) / 2}=1$, and

$$
\alpha^{\prime}=\left(1-\left\{\frac{k a}{n}\right\}\right) \pi, \quad \beta^{\prime}=\left(1-\left\{\frac{k b}{n}\right\}\right) \pi, \quad \gamma^{\prime}=\left(1-\left\{\frac{k c}{n}\right\}\right) \pi
$$

if $\varepsilon \cdot(-1)^{(k-1) / 2}=-1$, where $\varepsilon$ is the common value of $\varepsilon_{j}, j=1, \ldots, t$.

Note that by $4 \mid n$ we have $i=\zeta^{n / 4} \in \mathbb{Q}(\zeta)$ and $\phi(i)=\zeta^{k n / 4}=(-1)^{(k-1) / 2} i$. If we identify $\mathbb{R}^{2}$ with $\mathbb{C}$ then we find that for every $z=x+i y \in \mathbb{Q}(\zeta), \Phi(z)=$ $\phi(x)+i \phi(y)=\phi(z)$ if $(-1)^{(k-1) / 2}=1$, and $\Phi(z)=\overline{\phi(z)}$ if $(-1)^{(k-1) / 2}=-1$.

Clearly, $\Phi\left(V_{1}\right), \ldots, \Phi\left(V_{N}\right)$ are the vertices of a star polygon $R_{N}^{\prime}$. By the previous observation, the order of the vertices of $R_{N}^{\prime}$ is $1, \zeta^{k n / N}, \ldots, \zeta^{(N-1) k n / N}$ or $1, \zeta^{-k n / N}, \ldots, \zeta^{-(N-1) k n / N}$, depending on the sign of $(-1)^{(k-1) / 2}$.

Suppose $\{k / N\}<1 / 2$. Then the angles of $R_{N}^{\prime}$ at the vertices equal $(1-2\{k / N\}) \pi$, and the orientation of $R_{N}^{\prime}$ is positive or negative according to the sign of $(-1)^{(k-1) / 2}$.

Let $w(x ; P)$ denote the winding number of a closed polygon $P$ at a point $x \notin P$; that is, $w(x ; P)=(1 /(2 \pi i)) \int_{P} d z /(z-x)$. Since the boundary $\partial R_{N}^{\prime}$ of $R_{N}^{\prime}$ as an oriented cycle equals the sum of the boundaries $\partial \Delta_{j}^{\prime}$, we have

$$
w\left(x ; \partial R_{N}^{\prime}\right)=\sum_{j=1}^{t} w\left(x ; \partial \Delta_{j}^{\prime}\right) .
$$

If $x$ does not belong to the boundaries of $\Delta_{j}^{\prime}$, then we have either $w\left(x ; \partial \Delta_{j}^{\prime}\right)=\varepsilon$ or $w\left(x ; \partial \Delta_{j}^{\prime}\right)=0$ for every $j$. Therefore, if $w\left(x ; R_{N}^{\prime}\right)= \pm 1$, then $x$ belongs to exactly one of the triangles $\Delta_{j}^{\prime}$. Now, for each vertex $V_{j}^{\prime}, j=1, \ldots, N$, there is an angular domain $D_{j}$ of angle $(1-2\{k / N\}) \pi$, and there is a neighbourhood $U_{j}$ of $V_{j}^{\prime}$ such that $w\left(x ; R_{N}^{\prime}\right)=(-1)^{(k-1) / 2}$ if $x \in U_{j} \cap D_{j}$ and $w\left(x ; R_{N}^{\prime}\right)=0$ if $x \in U_{j} \backslash D_{j}$. This implies that $\varepsilon=(-1)^{(k-1) / 2}$, the triangles having a vertex at $V_{j}^{\prime}$ are nonoverlapping, and their union in $U_{j}$ equals $U_{j} \cap D_{j}$. Therefore, the angles $\alpha^{\prime}, \beta^{\prime}, \gamma^{\prime}$ are given by (6), and thus (3) and (4) hold. This proves the theorem in the case when $4 N \mid n$.

In the general case we put $m=4 N n$. Then we have $\alpha=(4 N a / m) \pi, \beta=$ $(4 N b / m) \pi, \gamma=(4 N c / m) \pi$. 
Let $k$ be prime to $n N$, and suppose $\{k / N\}<1 / 2$. Then $k+s n N$ is prime to $m$ for a suitable $s$ by Proposition 1.15. Since $\{(k+s n N) / N\}=\{k / N\}<1 / 2$, and $\{(k+s n N) \cdot 4 N a / m\}=\{k a / n\}$, etc., we obtain (3) and (4).

\section{Proof of Theorem 1.3}

In the next two sections we write $\delta$ for $\delta_{N}$. By Theorem 1.2, the equation at each vertex of $R_{N}$ equals one of $\alpha=\delta, \beta=\delta, \gamma=\delta, \alpha+\beta=\delta, \alpha+\gamma=\delta, \beta+\gamma=\delta, 2 \alpha=\delta$, $2 \beta=\delta, 2 \gamma=\delta$.

First suppose that $\alpha=\delta$ is one of the equations. If $\beta=\delta$ is another, then $\alpha+\beta<\pi$ gives $2 \delta<\pi, 2(N-2) / N<1$, and $N<4$, which is impossible. We have the same conclusion if $\gamma=\delta$. It is clear that $\alpha+\beta=\delta$ or $\alpha+\gamma=\delta$ is impossible. If $\beta+\gamma=\delta$, then $2 \delta=\alpha+\beta+\gamma=\pi, \delta=\pi / 2$, and $N=4$, which is impossible. Clearly, $2 \alpha=\delta$ is impossible. If $2 \beta=\delta$, then $\alpha+\beta<\pi$ gives $\alpha+\beta=3 \delta / 2=3 \pi / 2-3 \pi / N<\pi$ and, $N<6$, which is impossible. We have the same conclusion if $2 \gamma=\delta$. We find that if $\alpha=\delta$ is one of the equations, then each of the equations is $\alpha=\delta$, and we have (i). Therefore, we may assume that the equation at each vertex $V_{j}$ equals one of $\alpha+\beta=\delta$, etc., $2 \alpha=\delta$, etc.

Suppose that $\alpha+\beta=\delta$ is one of the equations. If $\alpha+\gamma=\delta$ is another, then $\beta=\gamma, \alpha=\pi-2 \beta, \delta=\pi-\beta, \beta=\gamma=2 \pi / N, \alpha=(N-4) \pi / N$. Let

$$
k= \begin{cases}(N-1) / 2 & \text { if } N \text { is odd } \\ N / 2-1 & \text { if } 4 \mid N \\ N / 2-2 & \text { if } N \equiv 2(\bmod 4)\end{cases}
$$

Then $\operatorname{gcd}(k, N)=1$ and $0<k<N / 2$. By Lemma 1.10, this implies that (3) holds, hence $4 k / N=\{2 k / N\}+\{2 k / N\}<1$ and $k<N / 4$. If $N$ is odd, then this implies $(N-1) / 2<N / 4$, which is impossible. If $4 \mid N$, then $N / 2-1<N / 4$ is also impossible. If $N \equiv 2(\bmod 4)$, then we get $N / 2-2<N / 4, N<8, N=6$, which is excluded. We have the same conclusion if $\beta+\gamma=\delta$. If $2 \gamma=\delta$ is another equation, then $\pi=\alpha+\beta+\gamma=3 \delta / 2,3(N-2) /(2 N)=1$, and $N=6$, which is impossible.

We find that if $\alpha+\beta=\delta$ is one of the equations, then either each of the equations is $\alpha+\beta=\delta$, that is, (ii) holds, or each of the other equations is one of $\alpha+\beta=\delta$, $2 \alpha=\delta$ and $2 \beta=\delta$, and at least one of $2 \alpha=\delta$ and $2 \beta=\delta$ must occur. Then we have $\alpha=\beta=\delta / 2$, and the tiles are isosceles. It is easy to check that in this case we can exchange the labels of the angles $\alpha$ and $\beta$ in some of the tiles so that each equation at the vertices becomes $\alpha+\beta=\delta$, and thus (ii) holds. Therefore, we may assume that the equation at each vertex $V_{j}$ equals one of $2 \alpha=\delta, 2 \beta=\delta$, and $2 \gamma=\delta$. If all of these equations occur, then $\alpha=\beta=\gamma=\pi / 3, \delta=2 \pi / 3$, and $N=6$, which is excluded. If two of them, say $2 \alpha=\delta$ and, $2 \beta=\delta$ occur, then we have $\alpha=\beta=\delta / 2$, and the tiles are isosceles. Then, as above, we can exchange the labels of the angles $\alpha$ and $\beta$ in some of the tiles so that each equation at the vertices becomes $\alpha+\beta=\delta$, and thus (ii) holds. Finally, if only $2 \alpha=\delta$ occurs, then we have (iii). 


\section{Proof of Theorem 1.4}

We have $\alpha=\delta$ and $\beta+\gamma=2 \pi / N$. If $\beta=\gamma$, then we have $\beta=\gamma=\pi / N$, and we are done. Therefore, we may assume $\gamma>\beta$ by symmetry.

Let $\alpha=(a / n) \pi, \beta=(b / n) \pi, \gamma=(c / n) \pi$, where $a, b, c, n$ are positive integers such that $a+b+c=n$. Let $b / n=b_{2} / n_{2}$ and $c / n=c_{3} / n_{3}$, where $\operatorname{gcd}\left(b_{2}, n_{2}\right)=$ $\operatorname{gcd}\left(c_{3}, n_{3}\right)=1$. Then

$$
\frac{b_{2}}{n_{2}}=1-\frac{\alpha}{\pi}-\frac{\gamma}{\pi}=1-\frac{N-2}{N}-\frac{c_{3}}{n_{3}}=\frac{2}{N}-\frac{c_{3}}{n_{3}} .
$$

We apply Lemma 1.12 with $c_{3}$ in place of $a, n_{3}$ in place of $n$ and with $N^{\prime}=1$. Then we find that one of the following statements is true.

(i) There is a $k$ prime to $n_{3} N$ and such that $k \equiv 1(\bmod N)$ and $\left\{k c_{3} / n_{3}\right\} \geq 1 / 3$;

(ii) $N$ is odd and $n_{3} \mid 2 N$;

(iii) $N$ is even and $n_{3} \mid N$.

Suppose (i). Replacing $k$ by $k+j n_{3} N$ with a suitable $j$, we may assume that $k$ is prime to $n N$. By Lemma 1.10 we have (3) and $\{k a / n\}+\{k c / n\}<1$. Since $\{k a / n\}=\{k(N-2) / N\}=\{(N-2) / N\}=(N-2) / N$ and $\{k c / n\} \geq 1 / 3$, we have $(N-2) / N<2 / 3$ and $N<6$, which is impossible.

Next suppose (ii). Then (7) gives $n_{2} \mid 2 N$. Now we have $\left(b_{2} / n_{2}\right)+\left(c_{3} / n_{3}\right)=2 / N=$ $4 /(2 N)$, and thus we have $c_{3} / n_{3}=3 /(2 N)$ and $b_{2} / n_{2}=1 /(2 N)$ by $c_{3} / n_{3}>b_{2} / n_{2}$. Since $k=N+2$ is prime to $2 N$ and $\{k / N\}=2 / N<1 / 2$, it follows from (3) that

$$
\left\{\frac{3(N+2)}{2 N}\right\}+\left\{\frac{N+2}{2 N}\right\}<1,
$$

which is absurd.

Finally, suppose (iii). Then (7) gives $n_{2} \mid N$. Thus $b_{2} / n_{2} \geq 1 / N$ and $c_{3} / n_{3} \geq 1 / N$. Since $b_{2} / n_{2}+c_{3} / n_{3}=2 / N$ and $c_{3} / n_{3}>b_{2} / n_{2}$, this is impossible.

\section{Proof of Theorem 1.5}

We put

$$
N^{\prime}= \begin{cases}(N-1) / 2 & \text { if } N \text { is odd } \\ N / 2-1 & \text { if } 4 \mid N \\ N / 2-2 & \text { if } N \equiv 2(\bmod 4)\end{cases}
$$

Then $\operatorname{gcd}\left(N, N^{\prime}\right)=1$ and $\left\{N^{\prime} / N\right\}<1 / 2$.

Let $\alpha=a_{1} \pi / n_{1}$, where $\operatorname{gcd}\left(a_{1}, n_{1}\right)=1$. By Lemma 1.12 , at least one of the following statements is true:

(i) there exists a $k$ such that $k \equiv N^{\prime}(\bmod N), \operatorname{gcd}\left(k, n_{1} N\right)=1$, and $\left\{k a_{1} / n_{1}\right\} \geq$ $1 / 3$ 
(ii) $N$ is odd and $n_{1} \mid 2 N$; and

(iii) $N$ is even and $n_{1} \mid N$.

If (i) holds then we may assume that $k$ also satisfies $\operatorname{gcd}(k, n N)=1$. Indeed, if $k$ satisfies the conditions of (i), then so does $k+j n_{1} N$ for every $j$. Replacing $k$ by $k+j n_{1} N$ with a suitable $j$, we find that $\operatorname{gcd}(k, n N)=1$ will also hold. Therefore, by (4) of Lemma 1.10, we obtain

$$
\frac{1}{3} \leq\left\{\frac{k a_{1}}{n_{1}}\right\}=\left\{\frac{k a}{n}\right\}<1-2 \cdot\left\{\frac{k}{N}\right\}=1-2 \cdot \frac{N^{\prime}}{N}
$$

and $N^{\prime}<N / 3$, which is impossible by $N>10$.

Next suppose that $N$ is odd and $n_{1} \mid 2 N$ (case (ii)). Let $b / n=b_{2} / n_{2}$, where $\operatorname{gcd}\left(b_{2}, n_{2}\right)=1$. Since $\beta=(\alpha+\beta)-\alpha=(N-2) \pi / N-\alpha$, we have

$$
\frac{b_{2}}{n_{2}}=\frac{N-2}{N}-\frac{a_{1}}{n_{1}}
$$

and thus $n_{2} \mid 2 N$. Then $c_{3} / n_{3}=1-a_{1} / n_{2}-b_{2} / n_{2}$ gives $n_{3} \mid 2 N$. Therefore, we may assume $n=2 N$. We put $k=N^{\prime}$ if $N^{\prime}$ is odd and $k=N^{\prime}+N$ if $N^{\prime}$ is even. Then $\operatorname{gcd}(k, 2 N)=1$ and $\{k / N\}<1 / 2$, and thus (4) of Lemma 1.10 gives

$$
\begin{aligned}
\left\{\frac{k a}{2 N}\right\}+\left\{\frac{k b}{2 N}\right\} & =1-2\left\{\frac{k}{N}\right\}=1-2\left\{\frac{N^{\prime}}{N}\right\} \\
& =1-2 \cdot \frac{N^{\prime}}{N}=1-2 \cdot \frac{(N-1) / 2}{N}=\frac{1}{N}
\end{aligned}
$$

Since $\{k a /(2 N)\}$ and $\{k b /(2 N)\}$ are positive integer multiples of $1 /(2 N)$, (9) gives $\{k a /(2 N)\}=\{k b /(2 N)\}=1 /(2 N)$. Then $k a \equiv k b \equiv 1(\bmod 2 N) . \operatorname{By} \operatorname{gcd}(k, 2 N)$ $=1$ this implies $a \equiv b(\bmod 2 N), a=b$, and $\alpha=\beta=(1 / 2-1 / N) \pi$. That is, the statement of the theorem is true in this case.

Finally, suppose that $2 \mid N$ and $n_{1} \mid N$ (case (iii)). Then we may assume $n=N$ by (8). Then (4) of Lemma 1.10 gives

$$
\left\{\frac{N^{\prime} a}{N}\right\}+\left\{\frac{N^{\prime} b}{N}\right\}=1-2\left\{\frac{N^{\prime}}{N}\right\}=1-2 \cdot \frac{N^{\prime}}{N} .
$$

The value of $N^{\prime} / N$ is $1 / 2-1 / N$ if $4 \mid N$, and $1 / 2-2 / N$ if $N \equiv 2(\bmod 4)$. Thus $1-2 N^{\prime} / N$ equals either $2 / N$ or $4 / N$. Since $\left\{N^{\prime} a / N\right\}$ and $\left\{N^{\prime} b / N\right\}$ are positive integer multiples of $1 / N$, we have the following possibilities: $\left\{N^{\prime} a / N\right\}=\left\{N^{\prime} b / N\right\}=1 / N$, $\left\{N^{\prime} a / N\right\}=\left\{N^{\prime} b / N\right\}=2 / N$, or $\left\{\left\{N^{\prime} a / N\right\},\left\{N^{\prime} b / N\right\}\right\}=\{1 / N, 3 / N\}$. In the third case we may assume, by symmetry, that $\left\{N^{\prime} a / N\right\}=1 / N$.

In the first two cases we have $N^{\prime} a \equiv N^{\prime} b(\bmod N), a \equiv b(\bmod N), a=b$, $\alpha=\beta=(1 / 2-1 / N) \pi$, and we are done. Therefore, we may assume that $N \equiv 2$ $(\bmod 4)$ and $\left\{N^{\prime} a / N\right\}=1 / N$; that is, $N^{\prime} a \equiv 1(\bmod N)$. Since $N$ is even, $a$ must be 
odd. Now $N / 2$ is odd either, and thus $N a / 2 \equiv N / 2(\bmod N)$. Then, by $N^{\prime}=N / 2-2$ we obtain

$$
1 \equiv N^{\prime} a=\left(\frac{N}{2}-2\right) a \equiv \frac{N}{2}-2 a(\bmod N)
$$

and $2 a+1 \equiv N / 2(\bmod N)$. Since $0<a<N$, we have either $2 a+1=N / 2$, that is, $a=N / 4-1 / 2$, or $2 a+1=3 N / 2$, that is, $a=3 N / 4-1 / 2$. We consider these two cases separately.

Case 1: $a=N / 4-1 / 2$. By (i) of Lemma 1.14, if $N \geq 26$, then there is a $k$ such that $N / 4<k<N / 2, \operatorname{gcd}(k, N)=1$, and $k \equiv 3(\bmod 4)$. Then

$$
\left\{\frac{k a}{N}\right\}=\left\{\frac{k}{4}-\frac{k}{2 N}\right\}=\left\{\frac{3}{4}-\frac{k}{2 N}\right\}=\frac{3}{4}-\frac{k}{2 N}>\frac{1}{2} .
$$

On the other hand, (4) of Lemma 1.10 gives

$$
\left\{\frac{k a}{N}\right\}<1-2\left\{\frac{k}{N}\right\}<\frac{1}{2},
$$

a contradiction. If $N=14$, then $a=N / 4-1 / 2=3$ and $b=N-2-a=9$. In this case $k=3$ is prime to $14,3 / 14<1 / 2$, but

$$
\left\{\frac{k a}{N}\right\}+\left\{\frac{k b}{N}\right\}=\left\{\frac{9}{14}\right\}+\left\{\frac{27}{14}\right\}=\frac{22}{14}>1,
$$

a contradiction. If $N=18$, then $a=4$ and $b=12$. Then $k=7$ is prime to 18 , $7 / 18<1 / 2$, but

$$
\left\{\frac{k a}{N}\right\}+\left\{\frac{k b}{N}\right\}=\left\{\frac{28}{18}\right\}+\left\{\frac{84}{18}\right\}=\frac{22}{18}>1,
$$

a contradiction. If $N=22$, then $a=5$ and $b=15$. Then $k=7$ is prime to 22, $7 / 22<1 / 2$, but

$$
\left\{\frac{k a}{N}\right\}+\left\{\frac{k b}{N}\right\}=\left\{\frac{35}{22}\right\}+\left\{\frac{105}{22}\right\}=\frac{30}{22}>1,
$$

a contradiction. Therefore, the case $a=N / 4-1 / 2$ is impossible if $N>10$.

Case 2: $a=3 N / 4-1 / 2$. By (i) of Lemma 1.14, if $N \geq 26$, then there is a $k$ such that $N / 4<k<N / 2, \operatorname{gcd}(k, N)=1$, and $k \equiv 1(\bmod 4)$. Then

$$
\left\{\frac{k a}{N}\right\}=\left\{\frac{3 k}{4}-\frac{k}{2 N}\right\}=\left\{\frac{3}{4}-\frac{k}{2 N}\right\}=\frac{3}{4}-\frac{k}{2 N}>\frac{1}{2}>1-2 \cdot \frac{k}{N},
$$


a contradiction. If $N=14$, then $a=3 N / 4-1 / 2=10$ and $b=N-2-a=2$. In this case $k=5$ is prime to $14,5 / 14<1 / 2$, but

$$
\left\{\frac{k a}{N}\right\}+\left\{\frac{k b}{N}\right\}=\left\{\frac{50}{14}\right\}+\left\{\frac{10}{14}\right\}=\frac{18}{14}>1,
$$

a contradiction. If $N=18$, then $a=13$ and $b=3$. Then $k=5$ is prime to 18 , $5 / 18<1 / 2$, but

$$
\left\{\frac{k a}{N}\right\}+\left\{\frac{k b}{N}\right\}=\left\{\frac{65}{18}\right\}+\left\{\frac{15}{18}\right\}=\frac{26}{18}>1,
$$

a contradiction. If $N=22$, then $a=16$ and $b=4$. Then $k=5$ is prime to 22 , $5 / 22<1 / 2$, but

$$
\left\{\frac{k a}{N}\right\}+\left\{\frac{k b}{N}\right\}=\left\{\frac{80}{22}\right\}+\left\{\frac{20}{22}\right\}=\frac{34}{22}>1,
$$

a contradiction. Therefore, the case $a=3 N / 4-1 / 2$ is also impossible if $N>10$. This completes the proof of the theorem.

Note that in the proof of Theorem 1.8 we only used Theorems $1.3-1.5$. Therefore, as the proofs of Theorems 1.3-1.5 are completed, Theorem 1.8 is also proved (subject to the number theoretic Lemmas 1.12-1.14).

\section{Proof of Theorem 1.6}

By Theorem 1.8, we may assume that the tiling is irregular. By symmetry, we may assume $\beta \leq \gamma$. Then, by $\alpha / \pi=(N-2) /(2 N)$ we have

$$
\frac{\gamma}{\pi} \geq \frac{\beta+\gamma}{2 \pi}=\frac{\pi-\alpha}{2 \pi}=\frac{1}{2}-\left(\frac{1}{4}-\frac{1}{2 N}\right)=\frac{1}{4}+\frac{1}{2 N}>\frac{1}{4} .
$$

It follows that in every equation $p \alpha+q \beta+r \gamma=v \pi$ we have $r \leq 7$. Note that in every equation we have $p \leq 4$, as $\alpha>2 \pi / 5$ by $N>10$.

By the irregularity of the tiling, there exists an equation $p_{0} \alpha+q_{0} \beta+r_{0} \gamma=v_{0} \pi$ such that $v_{0}=1$ or 2 and $q_{0}<r_{0}$. We may assume $\min \left(p_{0}, q_{0}\right)=0$, since otherwise we turn to the equation $\left(p_{0}-m\right) \alpha+\left(q_{0}-m\right) \beta+\left(r_{0}-m\right) \gamma=\left(v_{0}-m\right) \pi$, where $m=\min \left(p_{0}, q_{0}\right)$. We have

$$
\left(p_{0}-q_{0}\right) \alpha+\left(r_{0}-q_{0}\right) \gamma=\left(v_{0}-q_{0}\right) \pi .
$$

We put

$$
u=p_{0}-q_{0}, \quad s=r_{0}-q_{0}, \quad t=2 v_{0}-p_{0}-q_{0} .
$$


Note that $-6 \leq u \leq 4$ and $1 \leq s \leq 7$ by $p_{0} \leq 4$ and $q_{0}<r_{0} \leq 7$. It is clear that $t \leq 4$. By $u \alpha+s \gamma=\left(v_{0}-q_{0}\right) \pi$ we obtain

$$
\begin{gathered}
\gamma=\frac{1}{s} \cdot\left[v_{0}-q_{0}-u\left(\frac{1}{2}-\frac{1}{N}\right)\right] \pi=\left[\frac{t}{2 s}+\frac{u}{s N}\right] \pi \\
\beta=\pi-\alpha-\gamma=\left[\left(\frac{1}{2}-\frac{t}{2 s}\right)+\frac{1}{N}-\frac{u}{s N}\right] \pi=\left[\frac{s-t}{2 s}-\frac{u-s}{s N}\right] \pi .
\end{gathered}
$$

Since $\beta>0$, we get $(s-t) N>2(u-s)=2\left(p_{0}-r_{0}\right) \geq-14$. Thus $s \geq t$, as $s-t<0$ would imply $N<14$. Next we show $s \leq 2 t$. Suppose $s>2 t$. Then

$$
0 \leq \frac{\gamma-\beta}{\pi}=\frac{2 t-s}{2 s}+\frac{2 u-s}{s N} \leq-\frac{1}{2 s}+\frac{2 u-s}{s N}
$$

hence $1 \leq 2(2 u-s) / N, N \leq 2(2 u-s) \leq 14$, which is impossible. Thus $s \leq 2 t$, which also implies $t \geq 1$.

Summing up: we have

$$
-6 \leq u \leq 4, \quad 1 \leq s \leq 7, \quad 1 \leq t \leq 4, \quad \text { and } \quad t \leq s \leq 2 t .
$$

So the angles $\beta$ and $\gamma$ can only have a finite number (more precisely, at most $11 \cdot 7 \cdot 4=$ 308) of possible values for every $N$. We show that if $N \geq 25$ and $N \neq 30,42$, then only $\gamma=\pi / 2$ and $\gamma=(1 / 2-1 / N) \pi$ are possible, as the other cases do not satisfy conditions $(\mathrm{K})$ and $(\mathrm{E})$. We distinguish between two cases.

Case I: $t=s$. By (11), this implies $2 v_{0}=p_{0}+r_{0}$. Then (12) and (13) give

$$
\beta=\frac{s-u}{s N} \cdot \pi \text { and } \gamma=\left(\frac{1}{2}+\frac{u}{s N}\right) \cdot \pi .
$$

Then $\beta>0$ gives $s>u$; that is, $r_{0}>p_{0}$. Thus the nonnegative integers $p_{0}, q_{0}, r_{0}, v_{0}$ satisfy the following conditions: $v_{0}=1$ or $2, \min \left(p_{0}, q_{0}\right)=0,2 v_{0}=p_{0}+r_{0}$, $p_{0}<r_{0}$, and $q_{0}<r_{0}$. It is easy to check that the quadruples $\left(p_{0}, q_{0}, r_{0}, v_{0}\right)$ satisfying these conditions are the following:

$$
\begin{array}{lll}
(0,0,2,1), & (0,1,2,1), \quad(0,0,4,2), \quad(0,1,4,2), \\
(0,2,4,2), & (0,3,4,2), & \text { and }(1,0,3,2) .
\end{array}
$$

The values of $(s-u) / s=\left(r_{0}-p_{0}\right) /\left(r_{0}-q_{0}\right)$ obtained in these cases are 1, 2, 4, $2 / 3$, and $4 / 3$. That is, the possible values of $\beta$ are $\pi / N, 2 \pi / N, 4 \pi / N, 2 \pi /(3 N)$, and $4 \pi /(3 N)$. The first two cases give the triples listed in the theorem.

Suppose $\beta=4 \pi / N$. Then $\gamma=(1 / 2-3 / N) \pi$. If $N \geq 43$, (ii) of Lemma 1.14 gives an integer $k$ such that $N / 6<k<N / 4$ and $\operatorname{gcd}(k, 2 N)=1$. Then $\{k b / n\}=$ $\{4 k / N\}>2 / 3$ and

$$
\left\{\frac{k c}{n}\right\}=\left\{\frac{k}{2}-\frac{3 k}{N}\right\}=\left\{\frac{1}{2}-\frac{3 k}{N}\right\}>\frac{3}{4},
$$


since $1 / 2<3 k / N<3 / 4$. Thus the triple $(\alpha, \beta, \gamma)$ does not satisfy condition $(\mathrm{K})$. It is easy to check that for every $25 \leq N<42$ the triple $(\alpha, \beta, \gamma)=((1 / 2-$ $1 / N) \pi, 4 \pi / N,(1 / 2-3 / N) \pi)$ does not satisfy condition $(\mathrm{K}) .{ }^{1}$ Therefore, the case $\beta=4 \pi / N$ is impossible if $N \geq 25$ and $N \neq 42$.

Next suppose $\beta=2 \pi /(3 N)$. Then $\gamma=(1 / 2+1 /(3 N)) \pi$. Let

$$
k= \begin{cases}N+1 \text { if } N \equiv 0 \text { or } 4(\bmod 6) \\ N+2 \text { if } N \equiv 3 \operatorname{or} 5(\bmod 6) \\ N+3 \text { if } N \equiv 2(\bmod 6) \\ N+4 \text { if } N \equiv 1(\bmod 6)\end{cases}
$$

Then $\operatorname{gcd}(k, 6 N)=1$, and $\{k / N\}<1 / 2$. We have $\{k b / n\}=\{2 k /(3 N)\}>2 / 3$ and

$$
\left\{\frac{k c}{n}\right\}=\left\{\frac{k}{2}+\frac{k}{3 N}\right\}=\left\{\frac{1}{2}+\frac{k}{3 N}\right\}>\frac{5}{6},
$$

since $1 / 3<\{k /(3 N)\}<1 / 2$. Thus $(\alpha, \beta, \gamma)$ does not satisfy condition $(\mathrm{K})$, and the case $\beta=2 \pi /(3 N)$ is impossible.

Finally, suppose $\beta=4 \pi /(3 N)$. Then $\gamma=1 / 2-1 /(3 N)$. We put $k=2 N+1$ if $N \not \equiv 1(\bmod 3)$, and $k=2 N+3$ if $N \equiv 1(\bmod 3)$. Then $\operatorname{gcd}(k, 6 N)=1$ and $\{k / N\}<1 / 2$. We have $\{k b / n\}=\{4 k /(3 N)\}>2 / 3$, since $8 / 3<4 k /(3 N)<3$. On the other hand,

$$
\left\{\frac{k c}{n}\right\}=\left\{\frac{k}{2}-\frac{k}{3 N}\right\}>\frac{3}{4}
$$

since $2 / 3<k /(3 N)<3 / 4$. Thus $(\alpha, \beta, \gamma)$ does not satisfy condition $(\mathrm{K})$. Therefore, the case $\beta=4 \pi /(3 N)$ is also impossible.

Case II: $t<s$. First suppose $N>500$. Then, by (13) we have

$$
\frac{\beta}{\pi}=\frac{s-t}{2 s}-\frac{u-s}{s N} \geq \frac{1}{2 s}-\frac{3}{s N}=\frac{N-6}{2 s N} \geq \frac{N-6}{14 N}>\frac{1}{15} .
$$

This implies that $q<30$ holds in every equation $p \alpha+q \beta+r \gamma=v \pi$. Consider any of these equations. Substituting (12) and (13) into $p \alpha+q \beta+r \gamma=v \pi$ we obtain

$$
p \cdot \frac{1}{2}+q\left(\frac{1}{2}-\frac{t}{2 s}\right)+r \cdot \frac{t}{2 s}+\frac{1}{N} \cdot\left[-p+q\left(1-\frac{u}{s}\right)+r \cdot \frac{u}{s}\right]=v
$$

and $A \cdot N=2 \cdot(-p s+q(s-u)+r u)$, where $A=2 s v-(p s+q(s-t)+r t)$. If $A \neq 0$, then

\footnotetext{
1 In this computation and also in the computer search needed in the proof of the next theorem I applied GNU Octave (https://www.gnu.org/software/octave/).
} 


$$
\begin{aligned}
N \leq 2 \cdot|-p s+q(s-u)+r u| & \leq 2 \cdot \max (q s+r u, p s+q u) \\
& \leq 2 \cdot(30 \cdot 7+7 \cdot 4)<500
\end{aligned}
$$

which is impossible. Therefore, we have $A=0$, hence $-p s+q(s-u)+r u=0$.

We proved that $-p s+q(s-u)+r u=0$ holds for every equation $p \alpha+q \beta+$ $r \gamma=v \pi$. Let $K$ denote the number of the tiles. Taking the sum of the equations $-p s+q(s-u)+r u=0$ we obtain $0=-(K-2 N) s+K(s-u)+K u=2 N s$, a contradiction. Therefore, case II is impossible if $N>500$.

If $N \leq 500$, then we check for every possible triple $(\alpha, \beta, \gamma)$ whether or not it satisfies conditions (K) and (E). If $N$ is given, then $\beta$ and $\gamma$ are determined by (13) and (12). As these formulae show, we may take $n=2 s N$. We check, for every choice of $u, s, t$ satisfying (14) and also $t<s$, whether or not (3) holds for every $k$ such that $\operatorname{gcd}(k, n N)=1$ and $\{k / N\}<1 / 2$.

A computer search shows that in the range $60<N \leq 500$ only $N=78$ produces triples $(\alpha, \beta, \gamma)$ satisfying condition (K). More precisely, for $N=78$ there is just one such triple, namely

$$
\left(\frac{38 \pi}{78}, \frac{17 \pi}{78}, \frac{23 \pi}{78}\right)
$$

However, the only equations $p \alpha+q \beta+r \gamma=v \pi$ in this case are $\alpha+\beta+\gamma=\pi$ and $2 \alpha+2 \beta+2 \gamma=2 \pi$. Thus (2) of condition (E) is not satisfied, since we have $p>q$ in the equations at the vertices of $R_{N}$. Thus the case $N=78$ cannot occur.

In the range $42<N \leq 60$ only $N=60$ produces triples $(\alpha, \beta, \gamma)$ satisfying condition $(\mathrm{K})$. For $N=60$ there are two such triples, namely

$$
\left(\frac{29 \pi}{60}, \frac{12 \pi}{60}, \frac{19 \pi}{60}\right) \text { and }\left(\frac{29 \pi}{60}, \frac{11 \pi}{60}, \frac{20 \pi}{60}\right)
$$

In the first case the only equations $p \alpha+q \beta+r \gamma=v \pi$ are $5 \beta=\pi, 10 \beta=2 \pi$, $\alpha+\beta+\gamma=\pi, 2 \alpha+2 \beta+2 \gamma=2 \pi$, and $\alpha+6 \beta+\gamma=2 \pi$. We can see that $q \geq r$ holds in each of these equations. Then (2) of condition (E) can hold only if the equations with $q>r$ do not occur in the tiling. The remaining equations are $\alpha+\beta+\gamma=\pi$ and $2 \alpha+2 \beta+2 \gamma=2 \pi$. Thus condition (E) is not satisfied, since we have $p>q$ in the equations at the vertices of $R_{N}$. So this case is impossible.

If $(\alpha, \beta, \gamma)$ equals the second triple of (16), then the equations $p \alpha+q \beta+r \gamma=v \pi$ are the following: $3 \gamma=\pi, 6 \gamma=2 \pi, \alpha+\beta+\gamma=\pi, 2 \alpha+2 \beta+2 \gamma=2 \pi$, $\alpha+\beta+4 \gamma=2 \pi$, and $3 \alpha+3 \beta=2 \pi$. We can see that $p=q$ holds in each of these equations. Since $p>q$ holds in the equations at the vertices of $R_{N}$, condition (E) is not satisfied, and this case is also impossible.

In the range $24<N \leq 42$ only $N=30$ and $N=42$ produce triples $(\alpha, \beta, \gamma)$ satisfying condition $(\mathrm{K})$. This completes the proof of the theorem. 


\section{Proof of Lemma 1.12}

We may assume $\operatorname{gcd}\left(N^{\prime}, 2 n N\right)=1$, since otherwise we replace $N^{\prime}$ by $N^{\prime}+j N$ with a suitable $j$. Suppose there is an odd prime $p$ such that $p \mid n$ and $p \nmid N$. Let $P$ denote the product of primes dividing $n$ and different from $p$. (Put $P=1$ if there is no such prime.) Let $N P a / n=M / m$, where $\operatorname{gcd}(M, m)=1$. Since $p \nmid N P a$ and $p \mid n$, we have $p \mid m$, and thus $m \geq p \geq 3$.

Let $s$ be such that $s M \equiv 1(\bmod m)$. Then $p \nmid s$, as $p \mid m$. Put $k_{i}=N^{\prime}+i s N P$ for every integer $i$. Then $k_{i}$ is not divisible by any prime divisor of $n N$ except perhaps $p$. But if $p \mid k_{i}$, then $p \nmid k_{i-1}, k_{i+1}$, since $p \nmid s N P$. Thus either $k_{i}$ is prime to $n N$ or both of $k_{i-1}, k_{i+1}$ are prime to $n N$. Now

$$
\frac{k_{i} a}{n}=\frac{N^{\prime} a}{n}+i \frac{s N P a}{n}=\frac{N^{\prime} a}{n}+i \frac{s M}{m} \equiv \frac{N^{\prime} a}{n}+\frac{i}{m} \quad(\bmod 1) .
$$

This implies, by $m \geq 3$, that there are two consecutive $i$ 's with $\left\{k_{i} a / n\right\} \geq 1 / 3$. For at least one of them, $k_{i}$ is prime to $n N$. We find that (i) holds.

Next suppose that every odd prime divisor of $n$ divides $N$. Suppose $N$ is odd. Then $k_{i}=N^{\prime}+2 i N$ is prime to $n N$ for every $i$. Now $k_{i} a / n=N^{\prime} a / n+2 i N / n$ and thus, if $n \backslash 2 N$, then for a suitable $i$ we have $\left\{k_{i} a / n\right\} \geq 1 / 2$. That is, we have either (i) or (ii) in this case.

If $N$ is even, then $k_{i}=N^{\prime}+i N$ is prime to $n N$ for every $i$. Since $k_{i} a / n=$ $N^{\prime} a / n+i N / n$, we find that if $n \backslash N$, then for a suitable $i$ we have $\left\{k_{i} a / n\right\} \geq 1 / 2$. That is, we have either (i) or (iii) in this case. This completes the proof.

\section{Proof of Lemma 1.13}

By symmetry, we may assume $p \geq q$. Let $a / n=a_{1} / n_{1}$ and $b / n=b_{2} / n_{2}$, where $\operatorname{gcd}\left(a_{1}, n_{1}\right)=\operatorname{gcd}\left(b_{2}, n_{2}\right)=1$. Applying (5) with $k=1$ we obtain

$$
\frac{p a+q b}{n}=\frac{N-2}{N}
$$

We consider three cases.

Case I: $N$ is odd. Then $N^{\prime}=(N-1) / 2$ is prime to $N$. Suppose $n_{1} \mid 2 N$. For a suitable $j, k_{1}=(N-1) / 2+j N$ is prime to $n N$. By (5) we obtain

$$
\frac{p}{2 N}+\varepsilon \leq \frac{p}{n_{1}}+\varepsilon \leq p\left\{\frac{k_{1} a_{1}}{n_{1}}\right\}+\varepsilon=p\left\{\frac{k_{1} a}{n}\right\}+\varepsilon=1-2\left\{\frac{k_{1}}{N}\right\}=\frac{1}{N},
$$

where $\varepsilon=q \cdot\left\{k_{1} b / n\right\}$. Therefore, we have $p \leq 2$. If $p=2$, then $\varepsilon=0$ and $q=0$. If $p \leq 1$, then $q \leq 1$, and we have $p+q \leq 2$ in both cases.

Therefore, we may assume that $n_{1}$ does not divide $2 N$. Then, applying Lemma 1.12, we find that (i) of Lemma 1.12 holds with $a_{1}$ and $n_{1}$ in place of $a$ and $n$. That is, there is a $k$ prime to $n_{1} N$ such that $k \equiv(N-1) / 2(\bmod N)$ and $\left\{k a_{1} / n_{1}\right\} \geq 1 / 3$. For a 
suitable $j, k_{2}=k+j n_{1} N$ will be prime to $n N$. Then (5) gives

$$
\frac{p}{3} \leq p\left\{\frac{k_{2} a_{1}}{n_{1}}\right\}=p\left\{\frac{k_{2} a}{n}\right\} \leq 1-2\left\{\frac{k_{2}}{N}\right\}=\frac{1}{N} \leq \frac{1}{3},
$$

$p \leq 1$, and we are done.

Case II: $4 \mid N$. Then $N^{\prime}=N / 2-1$ is prime to $N$. Suppose $n_{1} \mid N$. For a suitable $j$, $k_{3}=N / 2-1+j N$ is prime to $n N$. By (5) we obtain

$$
\frac{p}{N}+\varepsilon \leq \frac{p}{n_{1}}+\varepsilon \leq p\left\{\frac{k_{3} a_{1}}{n_{1}}\right\}+\varepsilon=1-2\left\{\frac{k_{3}}{N}\right\}=\frac{2}{N}
$$

where $\varepsilon=q \cdot\left\{k_{3} b / n\right\}$. From this we obtain $p+q \leq 2$ as in case I. If $n_{1} \backslash N$, then applying Lemma 1.12, we find that (i) of Lemma 1.12 holds with $a_{1}$ and $n_{1}$ in place of $a$ and $n$. That is, there is a $k$ prime to $n_{1} N$ such that $k \equiv N / 2-1(\bmod N)$ and $\left\{k a_{1} / n_{1}\right\} \geq 1 / 3$. For a suitable $j, k_{4}=k+j n_{1} N$ will be prime to $n N$. Then (5) gives

$$
\frac{p}{N}+\varepsilon \leq \frac{p}{3}+\varepsilon \leq p\left\{\frac{k_{4} a_{1}}{n_{1}}\right\}+\varepsilon=1-2\left\{\frac{k_{4}}{N}\right\}=\frac{2}{N},
$$

where $\varepsilon=q \cdot\left\{k_{4} b / n\right\}$. From this inequality we obtain $p+q \leq 2$ as above.

Case III: $N$ is even and $N / 2$ is odd. Then $N^{\prime}=N / 2-2$ is prime to $N$. Note that the first possible value of $N$ is 10 , as $N=6$ is excluded.

Case IIIa: $n_{1} \mid N$. Then $a / n=u / N$, where $0<u<N$ is an integer. Suppose $p \geq 2$. For a suitable $j, k_{5}=N / 2-2+j N$ is prime to $n N$. By (5) we obtain

$$
p\left\{\frac{k_{5} u}{N}\right\}+q\left\{\frac{k_{5} b}{n}\right\}=1-2\left\{\frac{k_{5}}{N}\right\}=\frac{4}{N} .
$$

Since $\left\{k_{5} u / N\right\}$ is a positive integer multiple of $1 / N$ and $p \geq 2$, we have $\left\{k_{5} u / N\right\}=$ $1 / N$ or $2 / N$. If $\left\{k_{5} u / N\right\}=2 / N$, then (18) gives $p=2, q=0$, and we are done. If $\left\{k_{5} u / N\right\}=1 / N$, then $k_{5} u \equiv 1(\bmod N), u$ is odd, $u N / 2 \equiv N / 2(\bmod N)$, $k_{5} u \equiv(N / 2-2) u \equiv N / 2-2 u \equiv 1(\bmod N)$, and $u \equiv(N / 2-1) / 2(\bmod N / 2)$. Now $2 u / N=2 a / n<1$ by (17), and thus $u=(N / 2-1) / 2=(N-2) / 4$.

Since $N \geq 10, N / 2-4$ is also prime to $N$. For a suitable $j, k_{6}=N / 2-4+j N$ is prime to $n N$. Then we have

$$
p\left\{\frac{k_{6} u}{N}\right\}+q\left\{\frac{k_{6} b}{n}\right\}=1-2\left\{\frac{k_{6}}{N}\right\}=\frac{8}{N},
$$

and thus $\left\{k_{6} u / N\right\} \leq 4 / N$. However, we have

$$
k_{6} u \equiv k_{5} u-2 u \equiv 1-2 u=1-\frac{N-2}{2} \equiv \frac{N+4}{2}(\bmod N)
$$


and $\left\{k_{6} u / N\right\}=(N+4) /(2 N)>1 / 2>4 / N$, a contradiction. Therefore, we have $p \leq 1$ and $p+q \leq 2$.

Case IIIb: $n_{1} \backslash N$. By Lemma 1.12, there is a $k$ prime to $n_{1} N$ with $k \equiv N / 2-2$ $(\bmod N)$ and $\left\{k a_{1} / n_{1}\right\} \geq 1 / 3$. For a suitable $j, k_{7}=k+j n_{1} N$ will be prime to $n N$. Then (5) gives

$$
\frac{p}{3} \leq p\left\{\frac{k_{7} a_{1}}{n_{1}}\right\}+q\left\{\frac{k_{7} b_{2}}{n_{2}}\right\}=1-2\left\{\frac{k_{7}}{N}\right\}=\frac{4}{N} \leq \frac{4}{10}<\frac{2}{3} .
$$

Thus $p \leq 1, p+q \leq 2$, and the proof is complete.

\section{Proof of Lemma 1.14}

Lemma 9.1 Let $u, m, N$ be integers such that $m, N>0$ and $\operatorname{gcd}(u, m)=1$. Let $p_{1}, \ldots, p_{s}$ be those primes that divide $N$ but not $m$. If $c>0$ and

$$
\frac{c N}{m}\left(1-\frac{1}{p_{1}}\right) \ldots\left(1-\frac{1}{p_{s}}\right) \geq 2^{s}
$$

then for every real number a there is an integer $k$ such that $a \leq k<a+c N$, $k \equiv u(\bmod \mathrm{m})$, and $\operatorname{gcd}(k, N)=1$.

Proof Let $A_{d}$ denote the set of integers $k$ such that $a \leq k<a+c N, k \equiv u(\bmod m)$, and $d \mid k$. If $\operatorname{gcd}(d, m)=1$, then there is a $j_{0}$ such that $j_{0} m \equiv-u(\bmod d)$, and then $A_{d}$ equals the set of numbers $u+j_{0} m+j m d$ such that $a \leq u+j_{0} m+j m d<a+c N$. Thus $\left|A_{d}\right|$ equals the number of integers $j$ with $b \leq j<b+c N /(m d)$, where $b=\left(a-u-j_{0} m\right) /(m d)$. Therefore, we have $\left|A_{d}\right|=c N /(m d)+\varepsilon_{d}$, where $\left|\varepsilon_{d}\right|<1$.

If $S$ denotes the number of integers $k$ such that $a \leq k<a+c N, k \equiv u(\bmod m)$, and $\operatorname{gcd}(k, N)=1$, then

$$
\begin{aligned}
S & =\sum_{d \mid p_{1} \cdots p_{s}} \mu(d)\left|A_{d}\right|=\sum_{d \mid p_{1} \cdots p_{s}} \mu(d) \frac{c N}{m d}+\sum_{d \mid p_{1} \cdots p_{s}} \mu(d) \cdot \varepsilon_{d} \\
& >\frac{c N}{m}\left(1-\frac{1}{p_{1}}\right) \ldots\left(1-\frac{1}{p_{s}}\right)-2^{s} .
\end{aligned}
$$

If (19) is true, then $S>0$, which proves the lemma.

Proof of (i) of Lemma 1.14 Let $p_{1}, \ldots, p_{s}$ be the odd prime divisors of the even number $N$. By Lemma 9.1, statement (i) of Lemma 1.14 is true, if

$$
\frac{N}{16}\left(1-\frac{1}{p_{1}}\right) \cdots\left(1-\frac{1}{p_{s}}\right) \geq 2^{s} .
$$

If $s \geq 4$, then

$N\left(1-\frac{1}{p_{1}}\right) \cdots\left(1-\frac{1}{p_{s}}\right) \geq 2\left(p_{1}-1\right) \cdots\left(p_{s}-1\right) \geq 2 \cdot 2 \cdot 4 \cdot 6 \cdot 10^{s-3}>16 \cdot 2^{s}$, 
and thus the statement is true. Therefore, we may assume $s \leq 3$. If $N>480$, then

$$
\frac{N}{16}\left(1-\frac{1}{p_{1}}\right) \cdots\left(1-\frac{1}{p_{s}}\right)>\frac{480}{16} \cdot \frac{1}{2} \cdot \frac{2}{3} \cdot \frac{4}{5}=2^{3},
$$

and then the statement is true again. Finally, it is easy to check that for every even integer $N \in[26,480]$ there are integers $k, k^{\prime}$ with the required properties.

Proof of (ii) of Lemma 1.14 Let $p_{1}, \ldots, p_{s}$ be the odd prime divisors of $N$. Applying Lemma 9.1 with $m=2$ and $u=1$ we obtain that statement (ii) of Lemma 1.14 is true, if

$$
\frac{N}{24}\left(1-\frac{1}{p_{1}}\right) \cdots\left(1-\frac{1}{p_{s}}\right) \geq 2^{s} .
$$

If $s \geq 4$, then

$$
\begin{aligned}
N\left(1-\frac{1}{p_{1}}\right) \cdots\left(1-\frac{1}{p_{s}}\right) & \geq\left(p_{1}-1\right) \cdots\left(p_{s}-1\right) \\
& \geq 2 \cdot 4 \cdot 6 \cdot 10^{s-3}>24 \cdot 2^{s},
\end{aligned}
$$

and thus the statement is true. Therefore, we may assume $s \leq 3$. If $N>720$, then

$$
\frac{N}{24}\left(1-\frac{1}{p_{1}}\right) \cdots\left(1-\frac{1}{p_{s}}\right)>\frac{720}{24} \cdot \frac{1}{2} \cdot \frac{2}{3} \cdot \frac{4}{5}=2^{3},
$$

and then the statement is true again. Finally, it is easy to check that for every integer $N \in[43,720]$ there is an integer $k$ with the required properties.

Funding Open access funding provided by the Eötvös Loránd University.

Open Access This article is licensed under a Creative Commons Attribution 4.0 International License, which permits use, sharing, adaptation, distribution and reproduction in any medium or format, as long as you give appropriate credit to the original author(s) and the source, provide a link to the Creative Commons licence, and indicate if changes were made. The images or other third party material in this article are included in the article's Creative Commons licence, unless indicated otherwise in a credit line to the material. If material is not included in the article's Creative Commons licence and your intended use is not permitted by statutory regulation or exceeds the permitted use, you will need to obtain permission directly from the copyright holder. To view a copy of this licence, visit http://creativecommons.org/licenses/by/4.0/.

\section{References}

1. Frederickson, G.N.: Dissections. Plane \& Fancy. Cambridge University Press, Cambridge (1997)

2. Grünbaum, B., Shephard, G.C.: Tilings and Patterns. W.H. Freeman and Company, New York (1987)

3. Laczkovich, M.: Tilings of polygons with similar triangles. Combinatorica 10(3), 281-306 (1990)

4. Laczkovich, M.: Tilings of polygons with similar triangles. II. Discrete Comput. Geom. 19(3), 411-425 (1998) 
5. Laczkovich, M.: Tilings of convex polygons with congruent triangles. Discrete Comput. Geom. $\mathbf{4 8}(2)$, 330-372 (2012)

6. Laczkovich, M.: Tilings of the regular $N$-gon with triangles of angles $\pi / N, \pi / N,(N-2) \pi / N$ for $N=5,8,10$ and 12. Comput. Geom. 92, \# 101690101690 (2021)

7. Langford, C.D.: Tiling patterns for regular polygons. Math. Gazette 44(348), 105-110 (1960)

8. Szegedy, B.: Tilings of the square with similar right triangles. Combinatorica 21(1), 139-144 (2001)

Publisher's Note Springer Nature remains neutral with regard to jurisdictional claims in published maps and institutional affiliations. 